\title{
OCCUPATION TIME AND THE LEBESGUE MEASURE OF THE RANGE FOR A LEVY PROCESS
}

\author{
S. C. PORT
}

(Communicated by William D. Sudderth)

\begin{abstract}
We consider a Levy process on the line that is transient and with nonpolar one point sets. For $a>0$ let $N(a)$ be the total occupation time of $[0, a]$ and $R(a)$ the Lebesgue measure of the range of the process intersected with $[0, a]$. Whenever $[0, \infty)$ is a recurrent set we show $N(a) / E N(a)-$ $R(a) / E R(a)$ converges in the mean square to 0 as $a \rightarrow \infty$. This in turn is used to derive limit laws for $R(a) / E R(a)$ from those for $N(a) / E N(a)$.
\end{abstract}

1. Introduction. Throughout this paper $X_{t}$ will be a transient Levy process that is not a compound Poisson process starting at 0 . Let $T_{x}=\inf \left\{t>0: X_{t}=x\right\}$ be the hitting time of the singleton $\{x\}$. We will assume throughout that $\{0\}$ is not essentially polar, i.e. that $P_{x}\left(T_{0}<\infty\right)>0$ on a set of positive measure. Let $G(x, A)=\int_{0}^{\infty} P_{x}\left(X_{t} \in A\right) d t$. Under the above conditions $G(x, \cdot)$ is absolutely continuous with respect to Lebesgue measure and there is a version of the density $g(x)$ having the following properties: (i) $g$ is bounded. (ii) $g$ is continuous except perhaps at 0 where it may have a jump. (iii) $g(0)=g(0+) \wedge g(0-)$. Let $C=$ $[g(0+) \vee g(0-)]^{-1}$ and let $h(x)=P\left(T_{x}<\infty\right)$. Then $h(x)=C g(x)$. The above facts can be found in [1].

For $a>0$ let $N(a)$ be the total occupation time of $[0, a]$ and let $R(a)$ be the Lebesgue measure of the range of the process intersected with $[0, a]$. Observe that

$$
E R(a)=\int_{0}^{a} h(x) d x=C \int_{0}^{a} g(x) d x=C E N(a) .
$$

Our goal in this paper is to investigate the asymptotic behavior of $R(a)$ and $N(a)$ as $a \rightarrow \infty$.

Pruitt and Taylor [6] seem to have been the first to investigate the asymptotic behavior of $R(a)$ as $a \rightarrow \infty$. They examined the asymmetric Cauchy processes. If the asymmetry parameter $\beta$ is -1 (so the process has no positive jumps) then $[0, \infty)$ is a transient set and $R(a) \uparrow R(\infty)$ a.s. In all other cases $[0, \infty)$ is a recurrent set and $R(a) \uparrow \infty$ a.s. In this case Pruitt and Taylor [6] showed that

$$
E R(a) \sim k_{1} \frac{a}{\ln a}, \quad a \rightarrow \infty,
$$

for a specified constant $k_{1}$, and found the remarkable fact that

$$
R(a) \ln a / a \stackrel{D}{\rightarrow} R
$$

Received by the editors July 27, 1987.

1980 Mathematics Subject Classification (1985 Revision). Primary 60J30.

Key words and phrases. Levy process, I.D. process, occupation time, range. 
where $R$ or $R-1$ is geometrically distributed, according as $\beta<0$ or $\beta>0$. In [4] the asymptotic behavior of $N(a)$ was determined for the asymmetric Cauchy processes. If $\beta=-1, N(a) \uparrow N(\infty)<\infty$ a.s. For $\beta>-1, N(a) \ln a / a \stackrel{D}{\rightarrow}$ $(1 / C) R$. Hence for $\beta>-1$ we have the curious fact that $N(a) / E N(a)$ and $R(a) / E R(a)$ have the same limiting distribution.

Let $\tilde{X}_{t}$ be a stable process with exponent $\alpha$ and asymmetry parameter $\beta$. For $b \neq 0$, the process $X_{t}=\widetilde{X}_{t}+b t$ is the stable process with drift $b$. Suppose $\alpha<1$. Then as for the asymmetric Cauchy processes, $[0, \infty)$ is a transient set if $\beta=-1$. In all other cases $[0, \infty)$ is a recurrent set. It was shown in [4] that for $\beta>-1, E N(a) \sim k_{2} a^{\alpha}, a \rightarrow \infty$, for a specified numerical constant $k_{2}$, and that $N(a) / E N(a) \stackrel{D}{\rightarrow} N, a \rightarrow \infty$, where $N$ is the total occupation time of $[0,1]$ by the process $\widetilde{X}_{t}$.

One of the motivations for the present paper was to find the limiting distribution for $R(a) / E R(a)$ for the stable processes with drift, exponent $\alpha<1$, and $\beta>-1$. Another motivation was to understand why the limiting distributions of $R(a) / E R(a)$ and $N(a) / E N(a)$ were the same for the asymmetric Cauchy processes with $\beta>-1$. Remarkably, the following holds.

THEOREM 1. Suppose $[0, \infty)$ is a recurrent set. Then

$$
E\left[\frac{R(a)}{E R(a)}-\frac{N(a)}{E N(a)}\right]^{2} \rightarrow 0, \quad a \rightarrow \infty .
$$

If $[0, \infty)$ is a transient set $R(a) \uparrow R(\infty)$ and $N(a) \uparrow N(\infty), N(\infty)$ and $R(\infty)$ are $<\infty$ a.s., and generally there is no connection between the distributions of $R(\infty)$ and $N(\infty)$.

Theorem 1 shows that when $[0, \infty)$ is a recurrent set then $N(a) / E N(a)$ and $R(a) / E R(a)$ are asymptotically equal. This not only explains the equality of the limiting distributions for the asymmetric Cauchy processes with $\beta>-1$, but also yields a powerful tool for finding the limiting distributions for $R(a) / E R(a)$.

In general, it is usually quite difficult to find the limiting distribution of $R(a) / E R(a)$ by direct methods, even when considerable information is available about the asymptotic behavior of $g(x)$. In contrast, it is usually a routine application of the method of moments to determine the limiting distribution of $N(a) / E N(a)$ (provided it exists). See e.g. [4]. We will illustrate the use of Theorem 1 by finding the limiting distributions of $R(a) / E R(a)$ for the stable processes with drift and exponent $\alpha<1$, and by rederiving the Pruitt-Taylor result.

Let $X_{t}$ be a stable process with drift and exponent $\alpha<1$. Assume $\beta>-1$. Let

$$
k_{2}=\left[1+\beta^{2} \tan \left(\frac{\pi \alpha}{2}\right)\right]^{-1} \Gamma\left(\frac{1-\alpha}{\pi}\right) \sin \left(\frac{\pi \alpha}{2}\right) \alpha^{-1}(1+\beta) .
$$

Then it was shown in [4] that $E N(a) \sim k_{2} a^{\alpha}, a \rightarrow \infty$, and, as mentioned above, $N(a) / k_{2} a^{\alpha} \stackrel{D}{\rightarrow} N$. Using these facts, (1.1), and Theorem 1 , we can conclude the following.

THEOREM 2. For a stable process with drift and exponent $\alpha<1$ and $\beta>-1$

$$
R(a) / C k_{2} a^{\alpha} \stackrel{D}{\rightarrow} N .
$$


Note: An explicit formula for $C$ can be found in [4].

As a final illustration of the use of Theorem 1 let $X_{t}$ be an asymmetric Cauchy process. Let $Z$ be a geometrically distributed random variable with parameter $\rho=(1-\beta) /(1+\beta)$ if $\beta>0$ and with parameter $(1+\beta) /(1-\beta)$ if $\beta<0$. Again, by an easy application of the method of moments, it was shown in [4] that

$$
\frac{N(a)}{a / \ln a} \stackrel{D}{\longrightarrow} \frac{1}{C}(Z+1) \quad \text { if } \beta>0
$$

and

$$
\frac{N(a)}{a / \ln a} \stackrel{D}{\longrightarrow} \frac{Z}{C} \quad \text { if } \beta<0 .
$$

Using Theorem 1 we can deduce the result due to Pruitt and Taylor [6] that

$$
\frac{R(a)}{a / \ln a} \stackrel{D}{\longrightarrow}(Z+1) \quad \text { if } \beta>0 \text { and } \quad \frac{R(a)}{a / \ln a} \stackrel{D}{\longrightarrow} Z \quad \text { if } \beta<0 .
$$

The proof given here via Theorem 1 seems to be substantially simpler than those given by Pruitt and Taylor $[\mathbf{5}, \mathbf{6}]$.

To prove Theorem 1 we will need the renewal theorem for $g(x)$. Recall that a process is called type I transient except if it is transient with finite mean $b$. It is then called type II.

THEOREM 3. Assume the process is type I. Then $\lim _{|x| \rightarrow \infty} g(x)=0$. Assume the process is type II transient and $b>0$. Then $\lim _{x \rightarrow-\infty} g(x)=0$ and $\lim _{x \rightarrow \infty} g(x)=1 / b$.

Both the stable processes with drift and exponent $\alpha<1$ and the asymmetric Cauchy processes have infinite mean. It is only for Levy processes with infinite mean that there is any real problem with the asymptotic behavior of $N(a)$ and $R(a)$. If the process $X_{t}$ has finite mean things are quite simple. Suppose $E X_{t}=b t$. If $b<0$ then $[0, \infty)$ is a transient set since $X_{t} \rightarrow-\infty$ a.s. On the other hand if $b>0, X_{t} \rightarrow \infty$ a.s. and $[0, \infty)$ is recurrent. Theorem 3 shows that for $b>0, g(x) \rightarrow 1 / b$ as $x \rightarrow \infty$ and $g(x) \rightarrow 0$ as $x \rightarrow-\infty$. Using this fact an easy computation shows that $E_{0}(N(a) / a-1 / b)^{2} \rightarrow 0$. Thus by Theorem 1 $N(a) / E N(a)$ and $R(a) / E R(a)$ converge in $L_{2}$ to 1 . This can be strengthened to a.s. convergence. That $N(a) / E N(a) \rightarrow 1$ a.s. is a routine application of the strong law of large numbers as we shall see. To establish that $R(a) / E R(a) \rightarrow 1$ a.s. we will need to extend a result due to Kesten, Spitzer, and Whitman, for Brownian motion on $R^{d}$ to the Levy processes considered here. Since this fact actually holds in maximum generality we will take this opportunity to place the fact in the literature.

Let $\mathcal{G}$ be a second countable locally compact Abelian group and let $X_{t}$ be an I.D. process on $\mathcal{G}$ such that the smallest closed subgroup of $\mathcal{G}$ containing all of the possible points of $X_{t}$ (see p. 158 of [3] for the definition of a possible point) is $\mathcal{G}$, and assume $X_{t}$ is transient. Let $C_{B}(t)$ be the Haar measure of the set $\left\{x: x \in X_{s}+B\right.$ for some $\left.s \leq t\right\}$ where $B$ is a compact set.

For Brownian motion on $R^{d}, d \geq 3$, Kesten, Spitzer, and Whitman showed that $\lim _{t \rightarrow \infty} C_{B}(t) / t=C(B)$ with probability one, where $C(B)$ is the capacity of $B$. This result was never published, but an outline of the proof can be found in [2, p. 252]. The full proof is in W. W. Whitman's 1964 Cornell University thesis. 
Examination of the proof reveals that in fact the process $X_{t}$ is Brownian motion if used in only two ways. (1) $X_{t}$ is an I. D. process. (2) Let $T_{B}=\inf \left\{t>0: X_{t} \in\right.$ $B\}$. Then

$$
\frac{1}{t} \int P_{x}\left(T_{B} \leq t\right) d x \rightarrow C(B), \quad t \rightarrow \infty
$$

and

$$
\int P_{x}\left(T_{B} \leq t, X_{s} \notin B \text { for all } s>t\right) d x=t C(B) .
$$

In [3] a capacity theory was developed for all transient I. D. processes on $\mathcal{G}$ and the analogs of (1.3) and (1.4) were shown to be valid. ((1.3) is (11.3) of [3] and $(1.4)$ is (11.14) of [3]). Consequently, the same proof given for the Brownian motion case shows the following holds.

THEOREM 4. Let $C_{B}(t)$ be as above. Then $\lim _{t \rightarrow \infty} C_{B}(t) / t=C(B)$ with probability one.

We will use Theorem 4 for $B=\{0\}$ to show the following.

THEOREM 5. Let $X_{t}$ be a type II transient process on $R$ with mean $b>0$. Then $N(a) / a \rightarrow 1 / b$ with probability one and $R(a) / a \rightarrow C / b$ with probability one as $a \rightarrow \infty$. Also $N(a) / a$ and $R(a) / C a$ converge in $L_{2}$ to $1 / b$.

Theorems 1 and 5 apply with obvious modifications to the occupation time $N\left(a_{1}, a_{2}\right]$ of $\left(a_{1}, a_{2}\right]$ and the Lebesgue measure of the range intersected with $\left(a_{1}, a_{2}\right]$ as $a_{1} \rightarrow-\infty, a_{2} \rightarrow \infty$. We omit the details.

More generally, let $\mathcal{G}$ be a second countable locally compact Abelian group and $X_{t}$ a Levy process on $\mathcal{G}$ as described above. Assume $X_{t}$ is transient and that $\{0\}$ is not essentially polar. Let $A_{1} \subseteq A_{2} \subseteq \cdots, \bigcup_{n} A_{n}=A$. Assume $A_{i}$ are relatively compact and that $A$ is recurrent. Let $N\left(A_{i}\right)$ be the occupation time of $A_{i}$ and $R\left(A_{i}\right)$ the Haar measure of the range of the process intersected with $A_{i}$. Then the same arguments show that

$$
E\left(\frac{N\left(A_{i}\right)}{E N\left(A_{i}\right)}-\frac{R\left(A_{i}\right)}{E R\left(A_{i}\right)}\right)^{2} \rightarrow 0 .
$$

We omit the details.

\section{Proofs.}

PROOF OF THEOREM 3 . We will establish the assertions of Theorem 3 for a type II process with mean $b>0$. The same kind of argument can be used to establish the assertions of the theorem for a type I process. As usual, let $C$ be the capacity of a point. Equation (7.6) of [3] shows $h(x+y) \geq h(x) h(y)$ or equivalently,

$$
g(x+y) \geq C g(x) g(y)
$$

Let $a>0$ and $h>0$. Then (2.1) shows

$$
\int_{a}^{a+h} g(y) d y \geq C g(a) \int_{0}^{h} g(x) d x .
$$


Using the renewal theorem (Theorem 10.1 of [1]) we find from (2.2) that

$$
\varlimsup_{a \rightarrow \infty} g(a) \leq \frac{1}{b}\left[\frac{C}{h} \int_{0}^{h} g(x) d x\right]^{-1}
$$

In all cases when $b>0, g$ is left continuous at 0 and $g(0+)=C^{-1}$. (See [1].) Thus letting $h \downarrow 0$ in (2.3) we find

$$
\varlimsup_{a \rightarrow \infty} g(a) \leq \frac{1}{b} .
$$

Taking $x+y=a$ in (2.1) we find

$$
g(a) \geq C g(x) g(a-x) .
$$

Thus

$$
h g(a) \geq C \int_{a-h}^{a} g(x) g(a-x) d x .
$$

Since $g(0+)=C^{-1}$ we can, for any given $\varepsilon>0$, find $h>0$ such that $C g(y) \geq 1-\varepsilon$ if $0<y<h$. Thus for such $h$

$$
g(a) \geq \frac{1-\varepsilon}{h} \int_{a-h}^{a} g(x) d x .
$$

Using the renewal theorem again we find

$$
\varliminf_{a \rightarrow \infty} g(a) \geq \frac{1-\varepsilon}{b}
$$

It follows from (2.4) and (2.6) that $\lim _{a \rightarrow \infty} g(a)=1 / b$. The renewal theorem also shows

$$
\lim _{a \rightarrow-\infty} \int_{a}^{a+h} g(x) d x=\lim _{a \rightarrow-\infty} \int_{a-h}^{a} g(x) d x=0 .
$$

Using this fact the same kind of argument shows $\lim _{a \rightarrow-\infty} g(x)=0$.

PROOF OF THEOREM 1. Observe that

$$
E N(a)^{2}=2 \int_{0}^{a} \int_{0}^{a} g(x) g(y-x) d x d y .
$$

Now

$$
\begin{aligned}
E R(a)^{2} / C^{2}=\frac{1}{C^{2}} \int_{0}^{a} \int_{0}^{a} P\left(T_{x}<\infty, T_{y}<\infty\right) d x d y \\
\quad=\frac{1}{C^{2}}\left[\int_{0}^{a} \int_{0}^{a} P\left(T_{x}<T_{y}<\infty\right)+P\left(T_{y}<T_{x}<\infty\right)\right] d x d y \\
\quad=\frac{1}{C^{2}} \int_{0}^{a} \int_{0}^{a}\left[P\left(T_{x}<T_{y}\right) P_{x}\left(T_{y}<\infty\right)+P\left(T_{y}<T_{x}\right) P_{y}\left(T_{x}<\infty\right)\right] d x d y \\
\quad \leq \int_{0}^{a} \int_{0}^{a}[g(x) g(y-x)+g(y) g(x-y)] d x d y
\end{aligned}
$$

Thus

$$
E R(a)^{2} / C^{2} \leq E N(a)^{2}
$$


Now

Thus

$$
R(a) N(a)=\int_{0}^{\infty} d t \int_{0}^{a} d x 1_{\left[T_{x}<\infty\right]} 1_{[0, a]}\left(X_{t}\right)
$$

$$
E[R(a) N(a)]=\int_{0}^{\infty} d t \int_{0}^{a} d x P\left(T_{x}<\infty, 0 \leq X_{t} \leq a\right)
$$

But

$$
\begin{aligned}
P\left(T_{x}<\infty, 0<X_{t} \leq a\right)= & \int_{0}^{t} P\left(T_{x} \in d s\right) P_{x}\left(0 \leq X_{t-s} \leq a\right) \\
& +P\left(t<T_{x}<\infty, 0 \leq X_{t} \leq a\right) .
\end{aligned}
$$

Integrating (2.10) over $t$ we find

$$
\begin{aligned}
& \int_{0}^{\infty} P\left(T_{x}<\infty, 0 \leq X_{t} \leq a\right) d t \\
& \quad=P\left(T_{x}<\infty\right) \int_{0}^{a} g(y-x) d y+\int_{0}^{\infty} P\left(t<T_{x}<\infty, 0 \leq X_{t} \leq a\right) d t
\end{aligned}
$$

Now

$$
\begin{aligned}
\int_{0}^{\infty} P( & \left.t<T_{x}<\infty, X_{t} \in[0, a]\right) d t \\
= & \int_{0}^{\infty} \int_{0}^{a} P\left(T_{x}>t, X_{t} \in d y\right) P_{y}\left(T_{x}<\infty\right) d t
\end{aligned}
$$

Let $G_{\{x\}}(0, A)=\int_{0}^{\infty} P\left(T_{x}>t, X_{t} \in A\right) d t$ and let $G(x, A)=\int_{0}^{\infty} P_{x}\left(X_{t} \in A\right) d t$.

The first passage equation shows

$$
G_{\{x\}}(0, A)=G(0, A)-P\left(T_{x}<\infty\right) G(x, A) .
$$

Since $G(x, d y)=g(y-x) d y$ it follows that

$$
\begin{aligned}
\int_{0}^{a} \int_{0}^{\infty} & P\left(T_{x}>t, X_{t} \in d y\right) P_{y}\left(T_{x}<\infty\right) d t=\int_{0}^{a} G_{\{x\}}(0, d y) P_{y}\left(T_{x}<\infty\right) \\
& =\int_{0}^{a} g(y) P_{y}\left(T_{x}<\infty\right) d y-P\left(T_{x}<\infty\right) \int_{0}^{a} g(y-x) P_{y}\left(T_{x}<\infty\right) d y \\
& =C \int_{0}^{a}\left[g(y) g(x-y) d y-C^{2} g(x) g(y-x) g(x-y)\right] d y .
\end{aligned}
$$

Using (2.11) and (2.9) we find

$$
\begin{aligned}
E[R(a) N(a)]= & C \int_{0}^{a} \int_{0}^{a} g(x) g(y-x) d x d y \\
& +C \int_{0}^{a} \int_{0}^{a} g(y) g(x-y) d x d y \\
& -C^{2} \int_{0}^{a} \int_{0}^{a} g(x) g(y-x) g(x-y) d x d y
\end{aligned}
$$

Using (2.7), (2.8), and (2.12) we find

$$
E\left[\frac{R(a)}{C}-N(a)\right]^{2} \leq 2 C \int_{0}^{a} \int_{0}^{a} g(x) g(y-x) g(x-y) d x d y .
$$


Now (1.2) will hold provided we can show

$$
\lim _{a \rightarrow \infty}(E N(a))^{-2} \int_{0}^{a} \int_{0}^{a} g(x) g(y-x) g(x-y) d x d y=0 .
$$

The assumption that $[0, \infty)$ is a recurrent set implies that the process is either type I or type II with mean $b>0$. In either case $\lim _{x \rightarrow-\infty} g(x)=0$. Thus for given $\varepsilon>0$ we can find $\delta<\infty$ such that $g(-z) \leq \varepsilon$ for all $z \geq \delta$. Let $\psi(x)=\int_{0}^{x} g(z) g(-z) d z$ and $M=\sup _{x} g(x)<\infty$. Now

$$
\begin{gathered}
\int_{0}^{a} \int_{0}^{a} g(x) g(y-x) g(x-y) d x d y=\int_{0}^{a} g(a-x) \psi(x) d x+\int_{0}^{a} g(x) \psi(x) d x \\
\quad=\int_{0}^{\delta}[g(a-x)+g(x)] \psi(x) d x+\int_{\delta}^{a}[g(a-x)+g(x)] \psi(x) d x \\
\quad \leq M^{3} \delta^{2}+2 \varepsilon\left(\int_{0}^{a} g(x) d x\right)^{2}+2 M^{2} \delta \int_{0}^{a} g(x) d x .
\end{gathered}
$$

Thus

$$
\varlimsup_{a \rightarrow \infty}(E N(a))^{-2} \int_{0}^{a} \int_{0}^{a} g(x) g(y-x) g(x-y) d x d y \leq 2 \varepsilon .
$$

Hence (2.14) holds.

PROOF OF THEOREM 5. The strong law of large number shows $X_{t} / t \rightarrow b$ as $t \rightarrow \infty$ with probability one. Fix $\varepsilon>0$. Then $\exists t_{0}(\omega)$ such that for $t \geq t_{0}(\omega),(b-\varepsilon) t \leq X_{t}(\omega) \leq(b+\varepsilon) t$ for all $\omega$ except perhaps for $\omega$ in a set of probability 0 . Assume $b-\varepsilon>0$ and $a>(b+\varepsilon) t_{0}$.

$$
N(a) \geq \int_{t_{0}}^{a / b+\varepsilon} 1_{[0, a]}\left(X_{t}\right) d t=\frac{a}{b+\varepsilon}-t_{0}
$$

and

$$
N(a) \leq \int_{0}^{a / b-\varepsilon} 1_{[0, a]}\left(X_{t}\right) d t \leq \frac{a}{b-\varepsilon}
$$

except for $\omega$ in the exceptional set. Hence

$$
\frac{1}{b+\varepsilon} \leq \varliminf_{a \rightarrow \infty} \frac{N(a)}{a} \leq \varlimsup_{a \rightarrow \infty} \frac{N(a)}{a} \leq \frac{1}{b-\varepsilon} .
$$

Thus $N(a) / a \rightarrow 1 / b$ with probability one. Theorem 3 shows $E N(a)=\int_{0}^{a} g(x) d x$ $\sim a / b$ as $a \rightarrow \infty$.

Let $R_{t}$ be the Lebesgue measure of $\left\{x: x=X_{s}\right.$ for some $\left.s \leq t\right\}$. Then for $\omega$ not in the exceptional set

$$
R(a) \leq R_{t}
$$

for all $t \geq(b-\varepsilon) \wedge t_{0}$. Assume $a / b-\varepsilon>t_{0}$. Then $R(a) \leq R_{a / b-\varepsilon}$. For $B=\{0\},\left\{X_{s}+B, s \leq t\right\}=\left\{X_{s}, s \leq t\right\}$. Thus by Theorem 4

$$
\lim _{t \rightarrow \infty} R_{t} / t=C
$$

with probability one. Hence for $\omega$ not in some set of probability 0 .

$$
\varlimsup_{a \rightarrow \infty} \frac{R(a)}{a} \leq \lim _{a \rightarrow \infty} \frac{R_{a / b-\varepsilon}}{a}=\frac{C}{b-\varepsilon}
$$


Similarly

$$
\varliminf_{a \rightarrow \infty} \frac{R(a)}{a} \geq \lim _{a \rightarrow \infty} \frac{R_{a / b+\varepsilon}-R_{t_{0}}}{a}=\frac{C}{b+\varepsilon}
$$

except for $\omega$ in the exceptional set. Thus $\lim _{a \rightarrow \infty} R(a) / a=C / b$ with probability one. Since $0 \leq R(a) / a \leq 1, E R(a)^{2} /(C a)^{2} \rightarrow 1 / b^{2}$. Thus $R(a) / C a \stackrel{L_{2}}{\rightarrow} 1 / b$. Theorem 1 now shows that $N(a) / a \stackrel{L_{2}}{\rightarrow} 1 / b$.

\section{REFERENCES}

1. J. Bretagnolle, Résultats de Kesten sur les processus à accroisements independantes, Séminaire de Probabilités. V, Lecture Notes in Math., vol. 191, Springer, 1971, pp. 21-36.

2. K. Ito and J. P. McKean, Jr., Diffusion processes and their sample paths, Springer, 1965.

3. S. C. Port and C. J. Stone, Infinitely divisible processes and their potential theory. I, Ann. Inst. Fourier (Grenoble) 21 (1971), 157-275.

4. S. C. Port, Stable processes with drift on the line, Trans. Amer. Math. Soc. (to appear).

5. W. E. Pruitt and S. J. Taylor, The behavior of asymmetric Cauchy processes for large time, Ann. Probab. 11 (1983), 302-327.

6. __ Some sample path properties of the asymmetric Cauchy processes, Proc. Sympos. Pure Math., vol. 31, Amer. Math. Soc., Providence, R. I., 1977, pp. 111-123.

Department of Mathematics, University of California, Los Angeles, CaliFORNIA 90024 